\title{
H. pylori infection confers resistance to apoptosis via Brd4-dependent BIRC3 eRNA synthesis
}

\author{
Yanheng Chen', Donald Sheppard', Xingchen Dong ${ }^{1}$, Xiangming $\mathrm{Hu}^{2}$, Meihua $\mathrm{Chen}^{3}$, Ruichuan $\mathrm{Chen}^{3}$, \\ Jayati Chakrabarti, ${ }^{4,6}$, Yana Zavros ${ }^{4,6}$, Richard M. Peek Jr and Lin-Feng Chen (D)
}

\begin{abstract}
H. pylori infection is one of the leading causes of gastric cancer and the pathogenicity of $H$. pylori infection is associated with its ability to induce chronic inflammation and apoptosis resistance. While H. pylori infection-induced expression of pro-inflammatory cytokines for chronic inflammation is well studied, the molecular mechanism underlying the apoptosis resistance in infected cells is not well understood. In this study, we demonstrated that H. pylori infectioninduced apoptosis resistance in gastric epithelial cells triggered by Raptinal, a drug that directly activates caspase-3. This resistance resulted from the induction of CIAP2 (encoded by BIRC3) since depletion of BIRC3 by siRNA or inhibition of CIAP2 via BV6 reversed H. pylori-suppressed caspase-3 activation. The induction of CIAP2 was regulated by H. pyloriinduced BIRC3 eRNA synthesis. Depletion of BIRC3 eRNA decreased H. pylori-induced CIAP2 and reversed H. pylorisuppressed caspase-3 activation. Mechanistically, H. pylori stimulated the recruitment of bromodomain-containing factor Brd4 to the enhancer of BIRC3 and promoted BIRC3 eRNA and mRNA synthesis. Inhibition of Brd4 diminished the expression of BIRC3 eRNA and the anti-apoptotic response to H. pylori infection. Importantly, H. pylori isogenic cagAdeficient mutant failed to activate the synthesis of BIRC3 eRNA and the associated apoptosis resistance. Finally, in primary human gastric epithelial cells, H. pylori also induced resistance to Raptinal-triggered caspase-3 activation by activating the Brd4-dependent BIRC3 eRNA synthesis in a CagA-dependent manner. These results identify a novel function of Brd4 in $\mathrm{H}$. pylori-mediated apoptosis resistance via activating BIRC3 eRNA synthesis, suggesting that Brd4 could be a potential therapeutic target for $\mathrm{H}$. pylori-induced gastric cancer.
\end{abstract}

\section{Introduction}

Prevention of apoptosis by bacterial pathogens has emerged as a new trait of bacterial pathogenesis ${ }^{1}$. Accumulating evidence indicates that the ability to inhibit apoptosis during infection provides the bacterial pathogens with a survival advantage for the establishment of infection $^{1}$. H. pylori is a Gram-negative bacterium that colonizes the gastric epithelium, causing chronic gastric

\footnotetext{
Correspondence: Lin-Feng Chen (Ifchen@illinois.edu)

${ }^{1}$ Department of Biochemistry, University of Illinois at Urbana-Champaign,

Urbana 61801 IL, USA

${ }^{2}$ Fujian Key Laboratory for Translational Research in Cancer and

Neurodegenerative Diseases, Institute for Translational Medicine, School of

Basic Medical Sciences, Fujian Medical University, Fuzhou 350122, China

Full list of author information is available at the end of the article

These authors contributed equally: Yanheng Chen, Donald Sheppard

Edited by J.-E. Ricci
}

inflammations and other gastric diseases, including gastric cancer ${ }^{2}$. In addition to the induction of pro-inflammatory cytokines, infection-associated inhibition of apoptosis is one of the mechanisms by which $H$. pylori infection stimulates cell proliferation and induces gastric cancer ${ }^{2}$. The H. pylori cag pathogenicity island (cag PAI) encodes a type IV secretion system that injects the bacterial virulence factor CagA into host epithelial cells ${ }^{3}$. CagA has been demonstrated to be critical for $H$. pylori-associated gastric cancer since infection with CagA-positive $H$. pylori strains is associated with an increased risk for gastric cancer compared to infection with CagA-negative strains ${ }^{4,5}$. The role of CagA in the pro-inflammatory cytokine production has been heavily studied, its contribution to cell survival is much less clear. CagA has been shown to affect cell proliferation and survival independent of

\section{(c) The Author(s) 2020}

(c) (i) Open Access This article is licensed under a Creative Commons Attribution 4.0 International License, which permits use, sharing, adaptation, distribution and reproduction in any medium or format, as long as you give appropriate credit to the original author(s) and the source, provide a link to the Creative Commons license, and indicate if changes were made. The images or other third party material in this article are included in the article's Creative Commons license, unless indicated otherwise in a credit line to the material. If material is not included in the article's Creative Commons license and your intended use is not permitted by statutory regulation or exceeds the permitted use, you will need to obtain permission directly from the copyright holder. To view a copy of this license, visit http://creativecommons.org/licenses/by/4.0/. 
inflammation since over-expression of CagA in mice induces hyperplasia in the gastric mucosa and polyps in the glandular stomach without inducing inflammation ${ }^{6}$.

Cellular apoptosis is mediated by the extrinsic or the intrinsic apoptosis pathway. The extrinsic pathway is initiated by the ligation of death receptors with FasL (Fas ligand) or TRAIL (TNF-related apoptosis-inducing ligand) at the plasma membrane, leading to the activation of initiator caspase- $8^{7,8}$. On the other hand, the intrinsic pathway is initiated with the release of cytochrome $\mathrm{c}$ from the mitochondria to form apoptosomes for the activation of initiator caspase- $-9^{7,8}$. The activated initiator caspase- 8 or -9 in turn activates the downstream effector caspase- 3 or -7 , which uses the protease activity to degrade a wide array of cellular proteins or DNA and induces cellular apoptosis ${ }^{7,8}$. Many small molecules have been developed as therapeutics in cancer treatment to trigger extrinsic or intrinsic apoptosis pathways in cancer cells ${ }^{9}$. Among these apoptosis-inducing small molecules, Raptinal is able to rapidly induce cancer cell death by directly activating the effector caspase- 3 , bypassing the activation of initiator caspase- 8 and $-9^{10}$.

The inhibitor of apoptosis proteins (IAPs) are a family of structurally related proteins, including XIAP (X-linked IAP), cIAP1 (cellular inhibitor of apoptosis protein-1), cIAP2, and Survivin, that inhibit apoptosis by blocking the activation of effector caspases ${ }^{11}$. Modulating the expression and activity of IAPs contributes to the apoptosis resistance by certain bacterial pathogens ${ }^{1}$. For example, upregulation of cIAP1, cIAP2, and XIAP is required to maintain apoptosis resistance in Chlamydia trachomatis-infected epithelial cells ${ }^{12}$. Similarly, $N$. gonorrhoeae infection increases the NF-kB-dependent expression of cIAP1 and cIAP2 to inhibit apoptosis $^{13}$. Interestingly, H. pylori has also been shown to up-regulate cIAP2 expression in gastric epithelial cells and the gastric mucosa of $H$. pylori infected mice ${ }^{14,15}$. More importantly, cIAP2 is overexpressed in more than $70 \%$ of human gastric cancer tissues, and the expression levels of cIAP2 are higher in H. pylori-positive patient samples than in $H$. pylori-negative samples ${ }^{15,16}$. These studies highlight an important function of cIAP2 upregulation in $H$. pylori infection-mediated gastric diseases. However, the detailed mechanism for the upregulation of cIAP2 by $H$. pylori infection remains obscure.

The expression of IAPs has been reported to be regulated by the transcription factor NF- $\mathrm{kB}$, which is an important regulator of the genes involved in innate and adaptive immune responses and for the survival and proliferation of certain cell types ${ }^{17}$. NF- $\mathrm{KB}$ is critically involved in $H$. pylori-induced pro-inflammatory cytokine expression and upregulation of cIAP2 in response to H. pylori infection ${ }^{14,18,19}$. NF-kB-dependent inflammatory gene expression is facilitated by the binding of various transcription regulators to the enhancers or promoters of
NF- $\mathrm{KB}$ target genes ${ }^{20}$. Bromodomain-containing factor Brd4 has emerged as a key NF-kB co-activator by activating the positive transcription elongation factor $b$ (P-TEFb) complex, which phosphorylates serine 2 of RNA polymerase II (RNAPII) for the efficient transcription elongation of NF- $\mathrm{kB}$-dependent inflammatory genes ${ }^{21,22}$. In addition to its critical role in the synthesis of mRNA of inflammatory genes, emerging evidence indicates that Brd4 also facilitates NF- $\mathrm{KB}$ to stimulate the synthesis of enhancer RNA (eRNA) of inflammatory genes ${ }^{23,24}$. eRNAs are non-coding RNAs that are directed by enhancers and participate in enhancer function, affecting chromatin accessibility, chromatin looping, and the recruitment of transcription co-factors to the promoters $^{25}$. Importantly, eRNAs have been implicated in the regulation of inflammatory transcription networks ${ }^{24,26}$. We have recently shown that Brd4 was involved in both H. pylori-induced ILI mRNA transcription and ILI eRNA synthesis $^{18}$. However, whether Brd4-regulated eRNA synthesis is involved in the expression of cIAP2 in H. pylori-infected gastric epithelial cells remains to be determined.

To explore the mechanism by which $H$. pylori induces apoptosis resistance, we demonstrated that Brd4mediated BIRC3 eRNA synthesis was essential for H. pylori-induced BIRC3 mRNA and cIAP2 protein expression. Brd4 and NF- $\mathrm{kB}$ were recruited to the enhancer region of BIRC3 to regulate BIRC3 eRNA and mRNA synthesis in a CagA-dependent manner. In addition, $H$. pylori infection also induced apoptosis resistance by activating the expression of BIRC3 eRNA in primary human gastric epithelial cells.

\section{Results}

\section{H. pylori infection confers resistance to Raptinal-induced apoptosis}

To investigate the effect of $H$. pylori infection on apoptosis resistance in gastric epithelial cells, we first tested the ability of Raptinal to induce apoptosis in three different gastric epithelial cells, including AGS, MKN45, and MKN28. Raptinal has been shown to be able to induce apoptosis by activating caspase- 3 within $30 \mathrm{~min}$ at a concentration of $10 \mu \mathrm{M}^{10}$. Treatment with $10 \mu \mathrm{M}$ of Raptinal for $2 \mathrm{~h}$ induced the cleavage of pro-caspase- 3 into it's active form in all three cell lines (Fig. 1a). Compared to Raptinal, the chemotherapeutic agent Cisplatin acted much slower. The activated caspase- 3 was observed $24 \mathrm{~h}$ after $10-\mu \mathrm{M}$ Cisplatin treatment with no cleaved caspase- 3 appeared in $2 \mathrm{~h}$ (Fig. 1a and data not shown). These results suggest that Raptinal is a more effective apoptosis inducer than Cisplatin in gastric epithelial cells. Consistent with its ability to induce caspase- 3 activation, Raptinal suppressed the proliferation of these gastric cancer cells (Fig. 1b). 
A

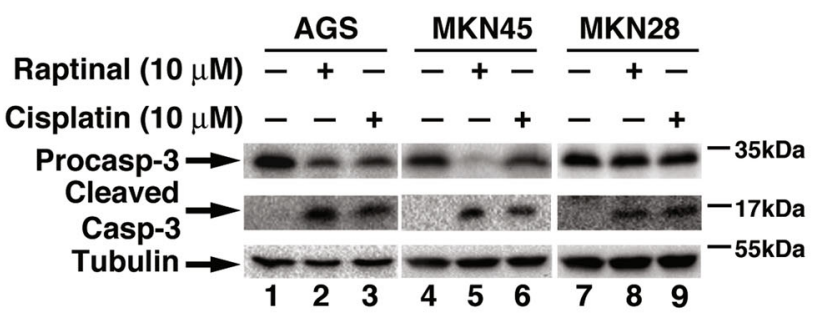

C

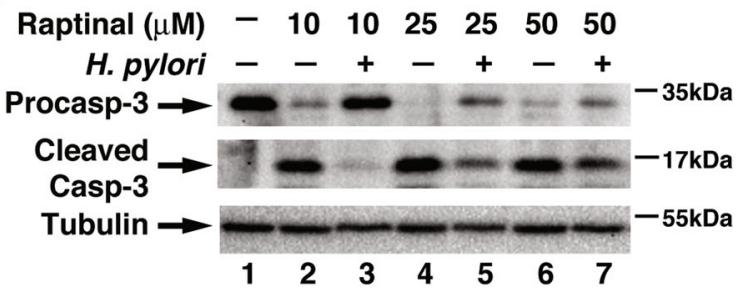

D
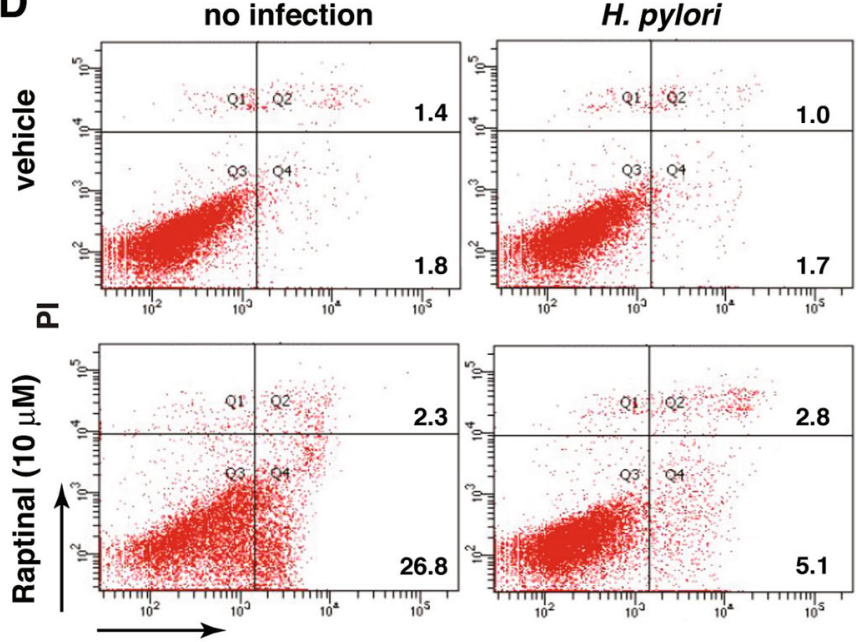

FITC-A
B

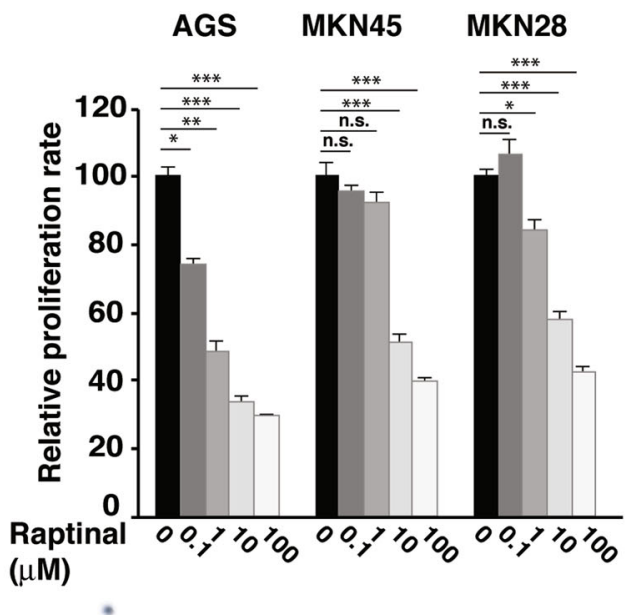

$\mathbf{E}$

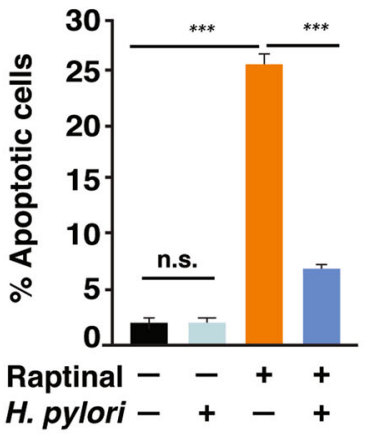

Fig. 1 H. pylori-infected gastric epithelial cells are resistant to Raptinal-induced apoptosis. a Indicated cell lines were treated with $10 \mu \mathrm{M}$ of Raptinal or Cisplatin for 2 or $24 \mathrm{~h}$, respectively. Cell lysates were analyzed by immunoblotting for indicated proteins. $\mathbf{b}$ Indicated gastric cancer cell lines were treated with $10 \mu \mathrm{M}$ of Raptinal for $72 \mathrm{~h}$, and cell proliferation was measured by A490 nm using the CellTiter $96^{\mathrm{R}}$ AQueous One Solution cell proliferation assay (MTS) (Promega). c AGS cells infected with H. pylori G27 for $1 \mathrm{~h}$ followed by treatment with indicated concentration of Raptinal for $2 \mathrm{~h}$. Cell lysates were analyzed by immunoblotting for indicated proteins. d AGS cells infected with $\mathrm{H}$. pylori G27 for $1 \mathrm{~h}$ followed by treatment with Raptinal $(10 \mu \mathrm{M})$ for $2 \mathrm{~h}$. Apoptosis was assessed by flow cytometry using Annexin V-FITC/PI double staining assay. e The percentage of apoptotic cells from (d). All data are shown as the mean \pm SD from three independent experiments. ${ }^{*} p<0.05,{ }^{* * *} p<0.001$, n.s.: not significant.

We next examined the effect of $H$. pylori on Raptinalinduced caspase-3 cleavage. H. pylori G27 infection inhibited the Raptinal-induced caspase-3 cleavage at $10 \mu \mathrm{M}$, and this ability of $H$. pylori was still partially exerted with increasing concentrations of Raptinal
(Fig. 1c). Since activation of caspase-3 is associated with cell apoptosis ${ }^{10}$, we then assessed the effect of $H$. pylori infection on Raptinal-induced apoptosis of gastric epithelial cells. Approximately 25\% of AGS cells underwent apoptosis $2 \mathrm{~h}$ after Raptinal treatment (Fig. 1d, e) and 
infection with $H$. pylori G27 efficiently reduced the percentage of Raptinal-induced apoptotic cells (Fig. 1d, e). Altogether, these data demonstrate that $H$. pylori suppressed Raptinal-induced apoptosis through inhibition of caspase-3 activation.

cIAP2 expression is required for $H$. pylori infection-induced apoptosis resistance in gastric epithelial cells

IAPs, including XIAP and CIAP1 and CIAP2, are the primary proteins that inhibits caspase activation in apoptosis $^{11}$. To understand the mechanism by which $H$. pylori infection induces apoptosis resistance, we measured the expression levels of these inhibitors of apoptosis in H. pylori infected AGS cells. Infection of AGS cells with H. pylori for $1 \mathrm{~h}$ dramatically increased the expression of BIRC3 (gene of cIAP2), but not the expression of BIRC2 (gene of cIAP1), BIRC4 (gene of XIAP), BIRC5 (gene of Survivin) and other apoptosis related genes (Figs. 2a, b and S1). The levels of cIAP2 also increased with $H$. pylori infection in a time-dependent manner (Fig. 2c). There was no significant change for the protein levels of cIAP1, XIAP, and Survivin during the course of infection (Fig. 2c).

To determine the role of cIAP2 in H. pylori infectioninduced apoptosis resistance, we treated the AGS cells with BV6, a known inhibitor of IAPs ${ }^{27}$, and examined the caspase- 3 activation induced by Raptinal. Similar to Fig. 1c, Raptinal-induced caspase-3 activation in AGS cells and the caspase- 3 activation was inhibited by $H$. pylori infection (Fig. 2d). However, this effect was reduced upon the addition of BV6 (Fig. 2d), indicating that inhibition of IAPs blocked H. pylori infection-induced apoptosis resistance. Consistently, more cells underwent apoptosis in $H$. pylori-infected AGS cells after BV6 treatment (Fig. 2e). These data suggest that IAPs might play a pivotal role in apoptosis resistance in response to $H$. pylori infection. Since $H$. pylori infection only induced the expression of cIAP2 but not XIAP and cIAP1 (Fig. 2c), cIAP2 was most likely responsible for the $H$. pylorimediated apoptosis resistance. We next knocked down the expression of cIAP2 using siRNA and observed increased caspase-3 cleavage after Raptinal treatment compared to the control cells upon $H$. pylori infection, similar to BV6-treated cells (Fig. 2f). Collectively, these data demonstrate that cIAP2 plays a critical role in H. pylori infection-induced resistance to apoptosis in gastric epithelial cells.

\section{H. pylori infection-induced resistance to apoptosis is mediated by BIRC3 eRNA}

Since $H$. pylori-induced cIAP2 expression is critical for apoptosis resistance, we next investigated how $H$. pylori stimulated the expression of BIRC3. We first determined the promoter and enhancer elements of BIRC3 located on Chromosome 11 in human cells. Promoters and enhancers are often defined by unique histone marks with H3K27Ac associated with the active transcription of enhancers and promoters, $\mathrm{H} 3 \mathrm{~K} 4 \mathrm{Me} 3$ associated with the promoters and H3K4me1 associated with active enhancers $^{28}$. We examined the available chromatin immunoprecipitation-sequencing (ChIP-seq) datasets for histone marks in various gastric cancer cell lines, and found that H3K27Ac was enriched around the $-10 \mathrm{~kb}$ region, upstream of TSS and the promoter region of BIRC3 (Fig. 3a). In AGS cells, H3K27Ac is largely enriched in the enhancer region from -11.45 to $-10.1 \mathrm{~kb}$ region but much less in the promoter region (Fig. 3a), suggesting that enhancers could be the major regulatory cis-element in the expression of BIRC3. Recent studies suggest that enhancer regulates gene expression via enhancer-derived eRNA, and we have previously showed that $H$. pylori induces eRNA synthesis for the efficient expression of inflammatory cytokine mRNAs such as $I L 1 A^{18,29}$. In order to determine whether H3K27Ac enriched enhancer regions regulates BIRC3 expression via eRNA, we examined the levels of eRNAs from several peaks containing enriched H3K27Ac, including -11.45 , -10.7 and $-10.1 \mathrm{k}$, with or without $H$. pylori infection (Fig. 3a). Of the three tested regions, $H$. pylori infection stimulated the expression of eRNAs significantly from $-10.7 \mathrm{k}$ region in AGS cells (Fig. $3 \mathrm{~b}$ ).

Since the function of eRNAs has largely been implicated in the regulation of its downstream mRNA, we next determined whether eRNAs from $-10.7 \mathrm{k}$ region was involved in the synthesis of BIRC3 mRNA in response to $H$. pylori infection. We designed two different siRNAs targeting the $-10.7 \mathrm{k}$ eRNAs to inhibit $H$. pylori-induced expression of eRNAs of BIRC3. These two siRNAs efficiently suppressed $H$. pylori-induced eRNA synthesis (Fig. 3c). Importantly, inhibition of $-10.7 \mathrm{k}$ eRNA not only suppressed the expression of BIRC3 mRNA but also the production of cIAP2 protein in response to $H$. pylori infection (Fig. 3d, e). We also assessed the effect of BIRC3 eRNA on the activation of caspase-3. Inhibition of BIRC3 eRNA synthesis by siRNAs reversed $H$. pylori-suppressed caspase3 activation (Fig. 3f). All together, these results indicate that BIRC3 gene expression is tightly regulated by BIRC3 eRNAs, and this eRNA synthesis is critical for the H. pylorimediated cIAP2 induction and caspase-3 inactivation.

\section{Brd4 is essential for BIRC3 eRNA synthesis and H. pylori infection-mediated apoptosis resistance}

Recent studies reveal that Brd4 is a key mediator in the eRNA synthesis by binding to the enhancers ${ }^{23}$. We have also demonstrated that Brd4 is essential for the synthesis of $I L 1$ eRNA in response to $H$. pylori infection ${ }^{18}$. We next determined whether Brd4 was involved in the synthesis of BIRC3 eRNA. We first treated AGS cells with JQ1, an inhibitor of Brd4 and other BET family proteins ${ }^{30,31}$ and 
A

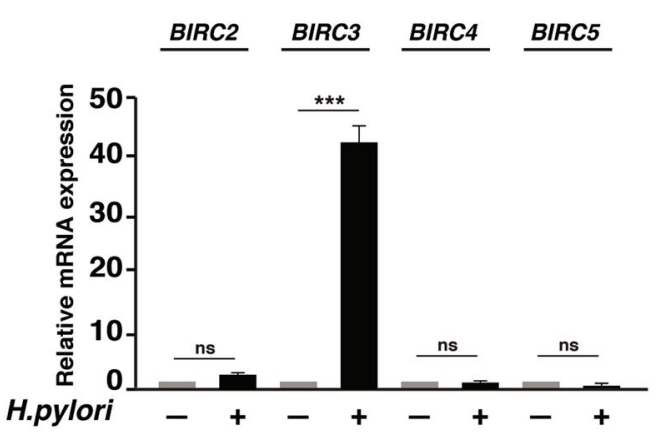

C

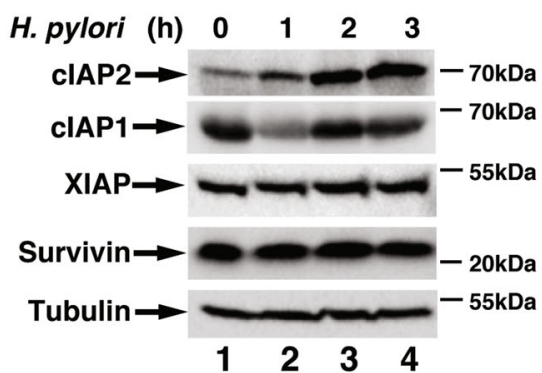

E

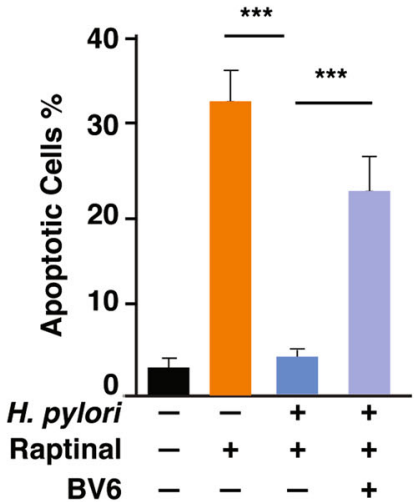

B

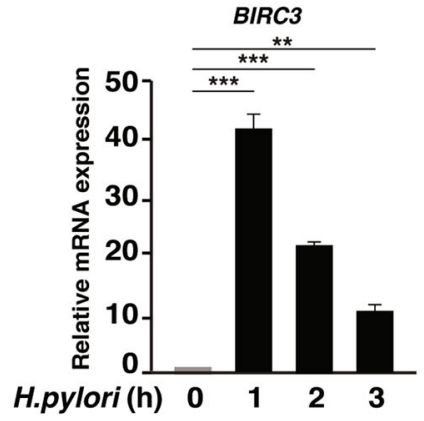

D

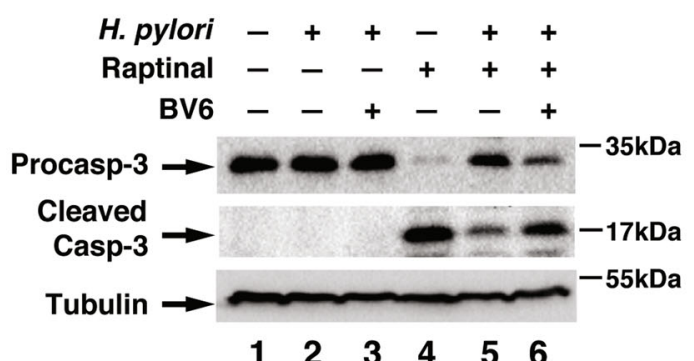

$\mathbf{F}$

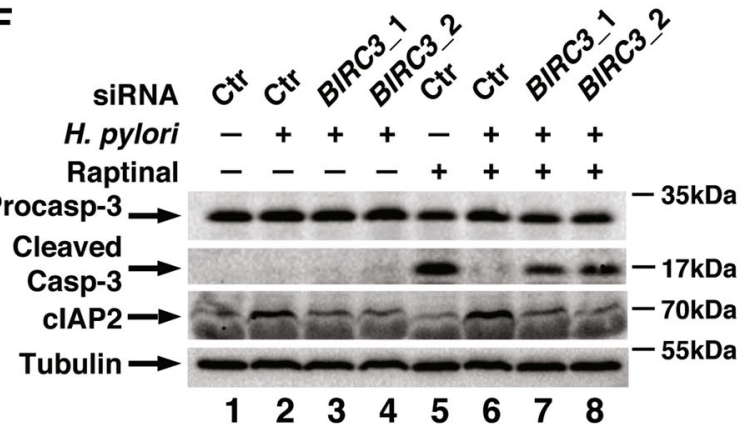

Fig. 2 cIAP2 is responsible for $\boldsymbol{H}$. pylori infection-mediated apoptosis resistance. a AGS cells were infected with $H$. pylori G27 for 1 h, and the expression of indicated genes was analyzed by qRT-PCR. b, c AGS cells were infected with H. pylori G27 for the indicated time points. mRNA levels of BIRC3 were analyzed by qRT-PCR (b) and cell lysates were analyzed by immunoblotting for indicated proteins (c). b AGS cells pre-treated with BV6 $(5 \mu \mathrm{M})$ for $1 \mathrm{~h}$ were infected with $\mathrm{H}$. pylori G27 for another $1 \mathrm{~h}$, followed by treatment with Raptinal $(10 \mu \mathrm{M})$ for $2 \mathrm{~h}$. Levels of indicated proteins were analyzed by immunoblotting, $n=3$. e Percentage of apoptotic cells from (c) was analyzed by flow cytometry with Annexin V-FITC/PI double staining. f AGS cells transfected with two different siRNAs against BIRC3 or control siRNA were infected with $\mathrm{H}$. pylori G27 for $1 \mathrm{~h}$, followed by treatment with Raptinal $(10 \mu \mathrm{M})$ for $2 \mathrm{~h}$. Levels of indicated proteins were analyzed by immunoblotting. All data are shown as the mean \pm SD from three independent experiments. ${ }^{* *} p<0.01,{ }^{* * *} p<0.001$, n.s.: not significant.

found that JQ1 inhibited both H. pylori-induced BIRC3 eRNA and mRNA synthesis (Fig. 4a, b). JQ1 also inhibited H. pylori-induced cIAP2 expression (Fig. 4c). To further confirm the involvement of Brd4 in the expression of BIRC3 eRNA and mRNA, we employed siRNAs to deplete Brd4 and other two BET proteins, including Brd2 and Brd3, and measured H. pylori-mediated BIRC3 expression. Depletion of Brd4 by siRNAs suppressed H. pylori-induced BIRC3 eRNA and mRNA expression (Fig. 4d, e). More importantly, depletion of Brd4 also inhibited $H$. pylori-induced cIAP2 expression (Fig. 4f). Further supporting the role of Brd4 in regulating 

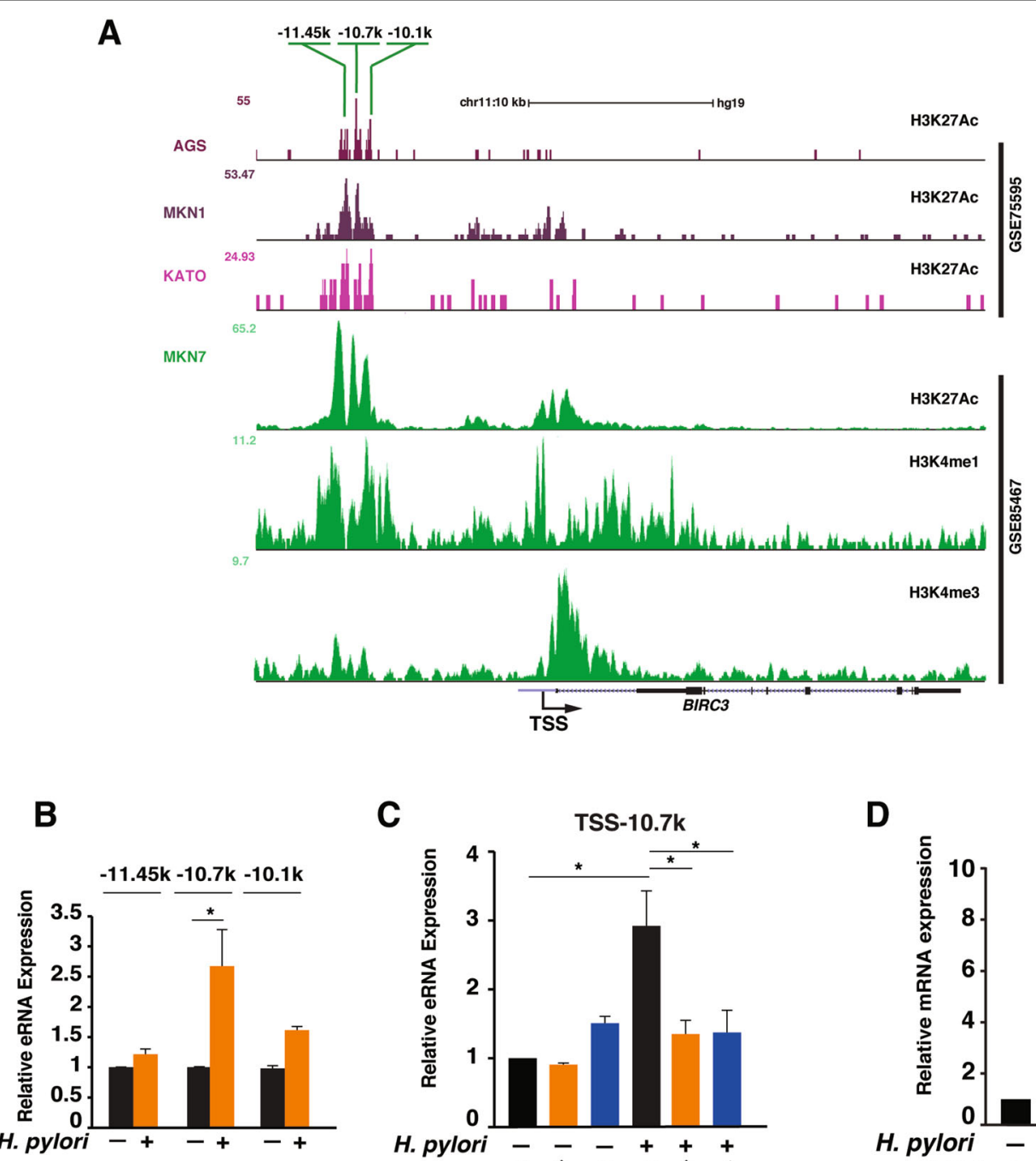

C

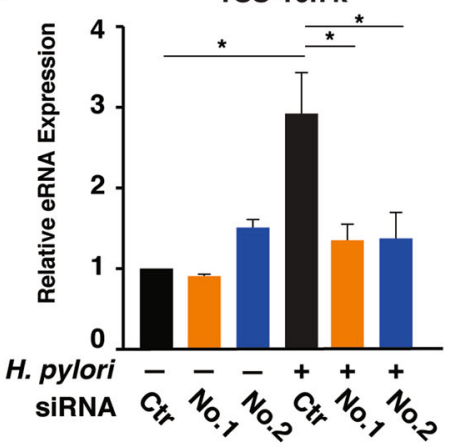

D

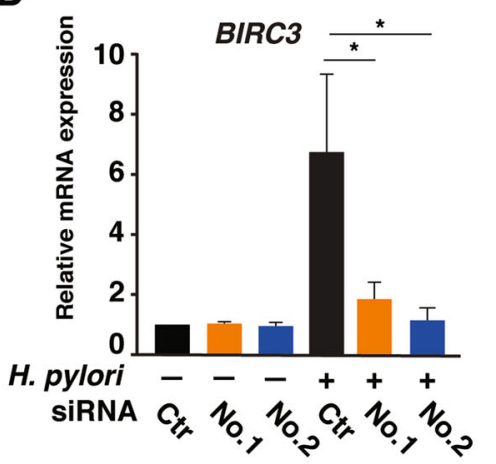

$\mathbf{E}$

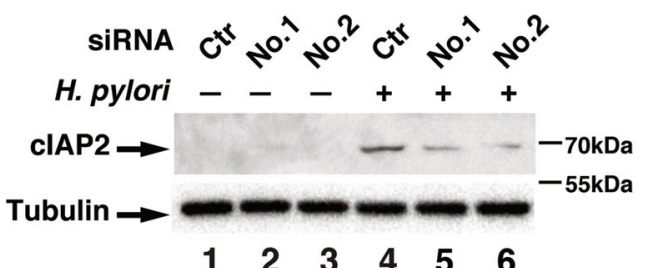

$\mathbf{F}$

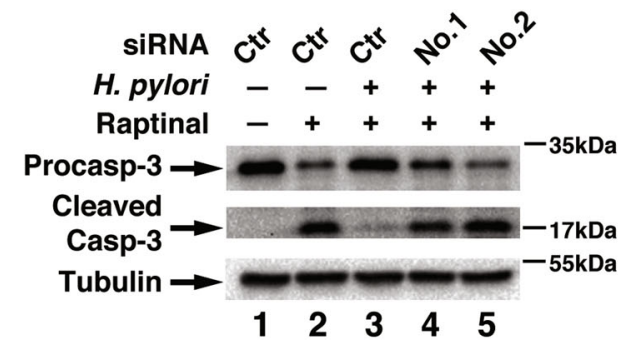

Fig. 3 H. pylori stimulates the expression of BIRC3 via eRNAs. a ChIP-seq profiles of two datasets (GSE75595 and GSE85467) for indicated histone marks of BIRC3 locus in various gastric cancer cell lines (AGS, MKN1, KATO, and MKN7). H3K27Ac peaks are marked with their relative distance from the BIRC3 TSS (Transcription Start Site). b AGS cells were infected with $\mathrm{H}$. pylori for $1 \mathrm{~h}$ and the expression of eRNAs from each potential enhancer were analyzed by qRT-PCR. $\mathbf{c}$, d AGS cells were transfected with two different siRNAs against eRNAs of BIRC3 from - $10.7 \mathrm{~kb}$, then infected with H. pylori G27 for $1 \mathrm{~h}$ and the expression of indicated BIRC3 eRNAs (c) and BIRC3 mRNA (d) were analyzed by qRT-PCR. e AGS cells transfected with siRNAs against BIRC3 eRNA as in (d) were infected with $\mathrm{H}$. pylori G27 for $2 \mathrm{~h}$ and the cell lysates were immunoblotted for indicated proteins. $\mathbf{f}$ AGS cells transfected with two siRNAs against BIRC3 eRNA as in (d) were infected with $\mathrm{H}$. pylori for $1 \mathrm{~h}$ followed by treatment with Raptinal (10 $\mu \mathrm{M})$ for $2 \mathrm{~h}$. Cell lysates were immunoblotted for indicated proteins. All data are shown as the mean \pm SD from three independent experiments. ${ }^{*} p<0.05$. 
A

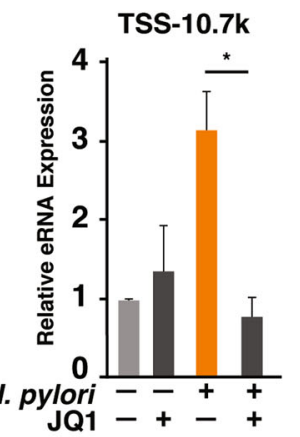

D

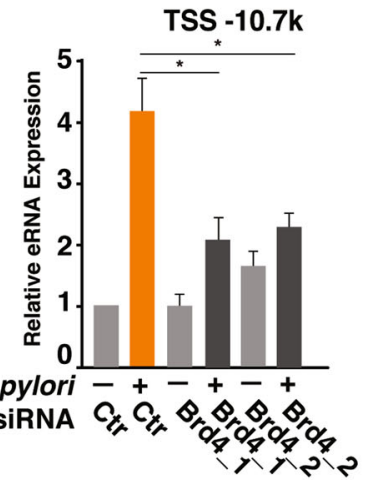

G

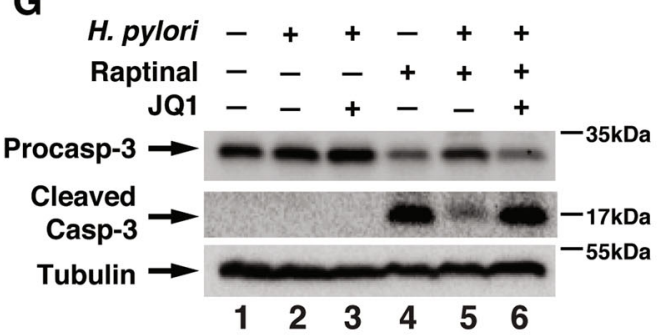

B
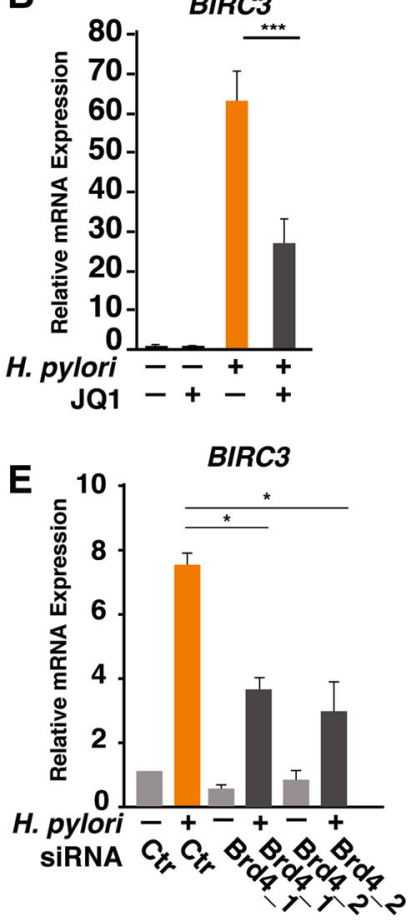

C

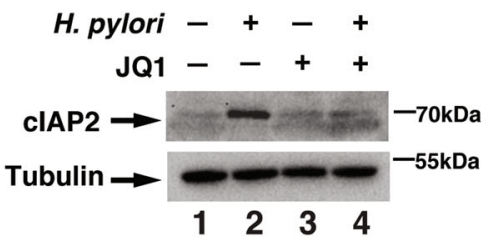

$\mathbf{F}$

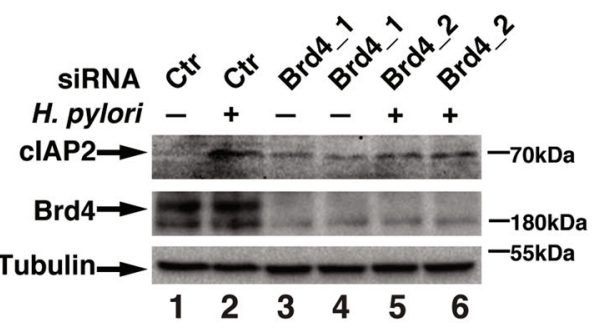

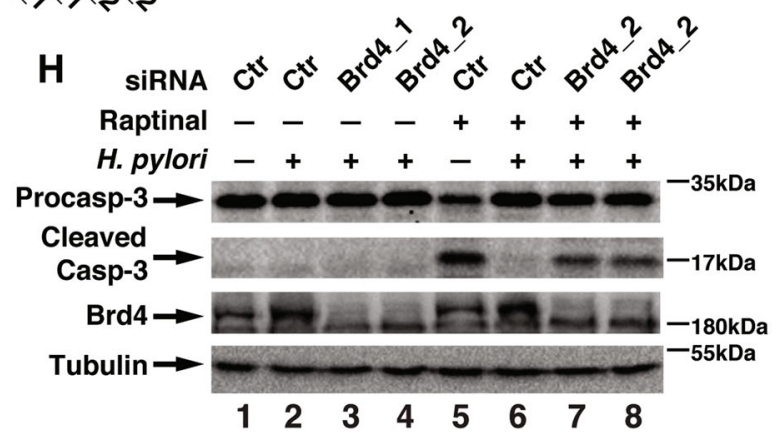

I
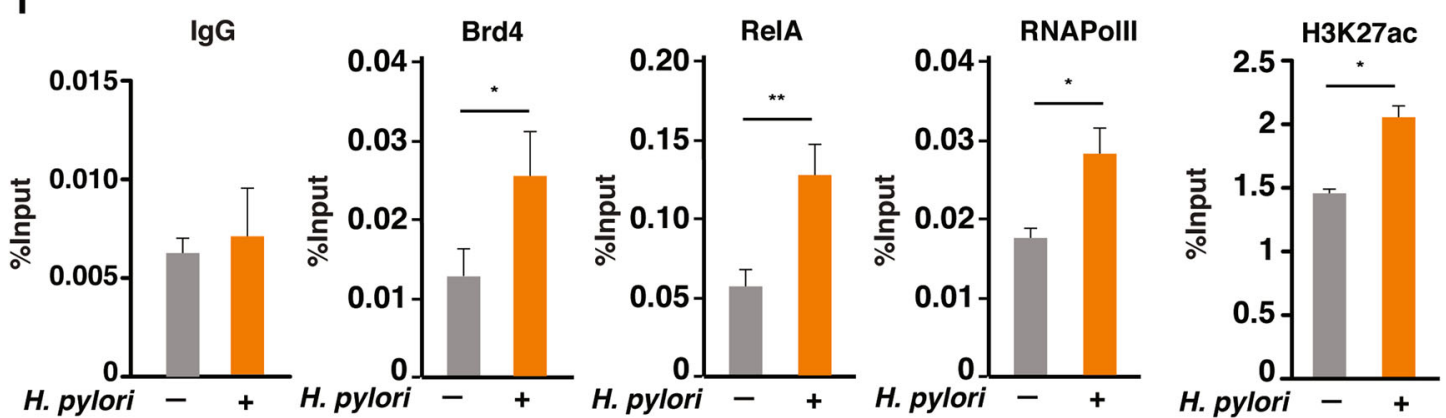

Fig. 4 Brd4 facilitates the synthesis of BIRC3 mRNA and eRNAs. a, b AGS cells were treated with or without $5 \mu \mathrm{M}$ JQ1 as indicated for $1 \mathrm{~h}$, followed by $H$. pylori infection for $1 \mathrm{~h}$. The expression of BIRC3 eRNAs (a) and mRNA (b) was analyzed by qRT-PCR. c AGS cells were treated with or without $5 \mu \mathrm{M}$ JQ1 for $1 \mathrm{~h}$, followed by $\mathrm{H}$. pylori infection for $2 \mathrm{~h}$. Cell lysates were immunoblotted for the indicated proteins. d, e AGS cells transfected with two different siRNAs against Brd4 were infected with $\mathrm{H}$. pylori for $1 \mathrm{~h}$. Expression of BIRC3 eRNAs (d) and mRNA (e) was analyzed by qRT-PCR. (f) AGS cells transfected with siRNAs against Brd4 as in (d, e) were infected with $\mathrm{H}$. pylori for $2 \mathrm{~h}$. Cell lysates were immunoblotted for indicated proteins. $\mathbf{g}$ AGS cells were pre-treated with or without $5 \mu \mathrm{M} \mathrm{JQ} 1$ for $1 \mathrm{~h}$ as indicated. Cells were then infected with $\mathrm{H}$. pylori for $1 \mathrm{~h}$ followed by treatment with Raptinal (10 $\mu \mathrm{M})$ for $2 \mathrm{~h}$. Cell lysates were immunoblotted for indicated proteins. $\mathbf{h}$ AGS cells transfected with siRNAs against Brd4 as in (d, e) were infected with or without H. pylori for $1 \mathrm{~h}$ followed by treatment with Raptinal $(10 \mu \mathrm{M})$ for $2 \mathrm{~h}$. Cell lysates were immunoblotted for indicated proteins. i AGS cells were infected with or without $\mathrm{H}$. pylori G27 for $1 \mathrm{~h}$. ChIP assays were performed using antibodies against Brd4, RelA, RNAPII and H3K27Ac and probed for the $-10.7 \mathrm{k}$ enhancer of BIRC3. All data are shown as the mean \pm SD from three independent experiments. ${ }^{*} p<0.05,{ }^{* *} p<0.01,{ }^{* * *} p<0.001$. 

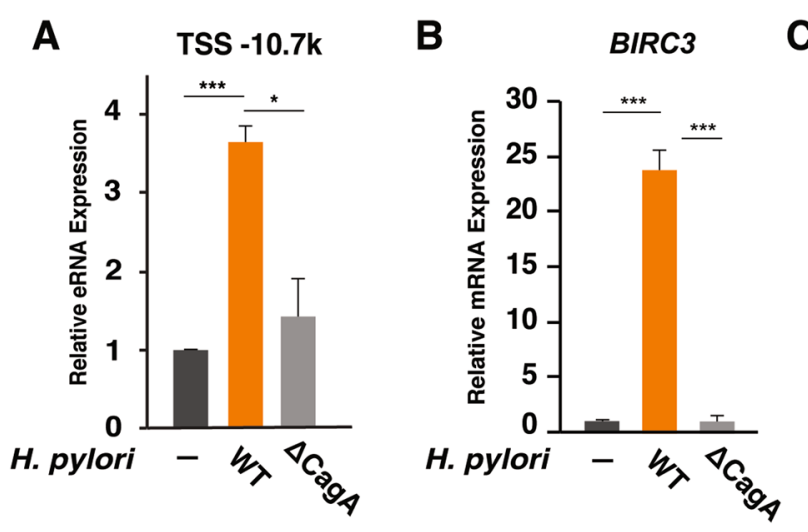

C

D
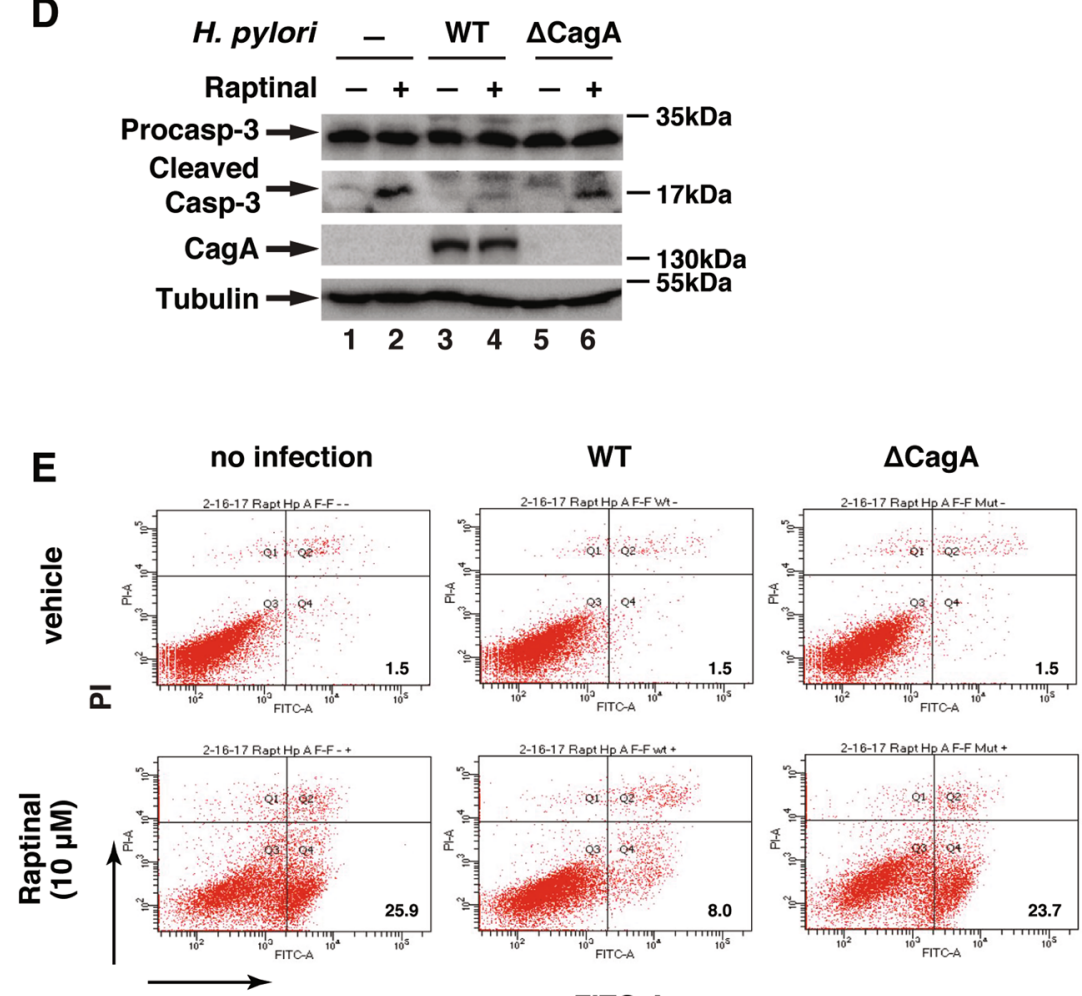

$\mathbf{F}$

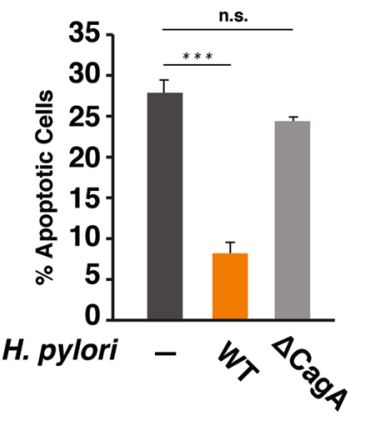

FITC-A

Fig. 5 CagA is essential for H. pylori-induced BIRC3 expression and apoptosis resistance. a, b AGS cells were infected with wild-type $H$. pylori G27 or the cagA-deficient isogenic mutant for 1 or $2 \mathrm{~h}$ for the expression of BIRC3 eRNA (a) or mRNA (b), respectively. Levels of BIRC3 eRNA and mRNA were analyzed by qRT-PCR. c AGS cells were infected with H. pylori G27 or cagA-deficient isogenic mutant for the indicated time points. Cell lysates were analyzed by immunoblotting for indicated proteins. d AGS cells were infected with WT H. pylori G27 or cagA-deficient isogenic mutant for $1 \mathrm{~h}$, followed by the treatment with Raptinal $(10 \mu \mathrm{M})$ for $2 \mathrm{~h}$. Cell lysates were immunoblotted for the indicated proteins. e AGS cells infected with WT H. pylori G27 or cagA-deficient isogenic mutant for $1 \mathrm{~h}$ followed by the treatment with Raptinal $(10 \mu \mathrm{M})$ for $2 \mathrm{~h}$. Apoptotic cells was analyzed by FACS with Annexin V-FITC/PI staining. $\mathbf{f}$ The percentage of apoptotic cells from (e) for Raptinal-treated samples. All data are shown as the mean \pm SD from three independent experiments. ${ }^{*} p<0.05,{ }^{* * *} p<0.001$, n.s.: not significant.

H. pylori-induced apoptosis resistance, H. pylori failed to suppress Raptinal-induced caspase-3 activation in JQ1 treated or Brd4 knockdown cells (Fig. 4g, h). However, depletion of Brd2 or Brd3 barely affected $H$. pyloriinduced BIRC3 mRNA and eRNA synthesis and cIAP2 expression (Figs. S2A-C) and did not affect H. pylori- induced apoptosis resistance (Fig. S2D). Collectively, these results demonstrate that $\mathrm{Brd} 4$ but not $\mathrm{Brd} 2$ or $\mathrm{Brd} 3$ is critically involved in $H$. pylori-induced cIAP2 induction and apoptosis resistance.

To further understand how Brd4 regulates BIRC3 eRNA synthesis, we employed ChIPs to determine whether Brd4 
is recruited to the enhancer region of BIRC3. Compared to uninfected cells, the binding of Brd4 to -10.7 enhancer region was significantly enhanced by $H$. pylori infection (Fig. 4i). NF-kB is known to assist the recruitment of Brd4 to the promoters or enhancers to activate CDK9 to facilitate RNAPII-dependent transcription elongation ${ }^{21}$. Consistently, the RelA subunit of NF- $\mathrm{kB}$ and RNAPII were also found to be enriched on the $-10.7 \mathrm{k}$ enhancer region after $H$. pylori infection (Fig. 4i). Similarly, H3K27Ac, the histone mark for active transcription, was also enriched in H. pylori infected cells (Fig. 4i). These ChIP results further demonstrate that $H$. pylori stimulates the recruitment of Brd4, likely through NF-кB, to the enhancer of BIRC3 for the RNAPII-mediated BIRC3 eRNA synthesis.

\section{H. pylori infection stimulates the expression of CIAP2 and apoptosis resistance in a CagA-dependent manner}

Translocation of $H$. pylori virulent factor CagA into host cells induces higher levels of cytokine production by activating NF- $\mathrm{BB}$ and $\mathrm{AP}-\mathrm{1}^{32}$. Due to the essential role of NF- $\kappa B$ in the expression of cIAP2 ${ }^{19,33}$, we next investigated whether CagA was involved in the BIRC3 eRNA and mRNA synthesis. Unlike WT $H$. pylori, cagA-deficient isogenic mutant failed to activate the expression of BIRC3 eRNA and mRNA (Fig. 5a, b). Examining the cellular levels of cIAP2 in $H$. pylori infected AGS cells revealed that the expression of cIAP2 was upregulated by the infection in a CagA-dependent manner since infection of AGS cells with WT but not the cagA-deficient H. pylori enhanced the expression levels of cIAP2 (Fig. 5c).

Since cIAP2 is critical for the H. pylori-mediated inhibition of caspase-3 activation (Fig. 2), we next examined the effect of CagA on Raptinal-induced caspase-3 activation. Infection of cells with WT but not the cagA-deficient mutant $H$. pylori suppressed Raptinal-induced caspase-3 activation (Fig. 5d). We also examined the effect of CagA on Raptinal-induced cell apoptosis. While WT H. pylori suppressed Raptinal-induced cell apoptosis, the cagAdeficient isogenic mutant barely affected Raptinal-induced cell apoptosis (Fig. 5e, f). Collectively, these findings demonstrate that H. pylori CagA is essential for the induction of cIAP2 expression and $H$. pylori-infectionmediated apoptosis resistance.

\section{H. pylori induces CIAP2 expression and inhibits apoptosis in primary human gastric epithelial cells}

Gastrointestinal organoids closely recapitulate the cellular complexity of the organ tissue from which they are derived to mimic intact tissue in vivo ${ }^{34,35}$. Culture of primary human- and mouse-derived gastric organoids has emerged as a novel approach to study the pathogenesis of H. pylori infection in a more physiological setting ${ }^{34,36-38}$. We next assessed the effect of $H$. pylori-mediated apoptosis resistance in human gastric organoids. We acquired organoids derived from primary tissues and cultured them to form a 2D gastric epithelial cell monolayer (Fig. 6a). Treatment of the gastric epithelial cell cells with Raptinal dramatically changed the cell morphology, indicating Raptinal-induced cell death (Fig. 6a, upper panels). However, infection of cells with $H$. pylori prevented Raptinal-induced morphology changes (Fig. 6a, middle panels). Conversely, infection with the cagA-deficient mutant failed to prevent Raptinal-induced cell morphology changes (Fig. 6a, lower panels), indicating CagA-dependent apoptosis resistance by $H$. pylori infection. We next measured $H$. pylori-stimulated BIRC3 eRNA and mRNA synthesis using these organoids. Similar to AGS cells, WT but not the cagA-deficient mutant H. pylori stimulated the expression of BIRC $3-10.7 \mathrm{k}$ eRNA and mRNA (Fig. 6b, c) and induced the cIAP2 expression (Fig. 6d). Furthermore, Raptinal also effectively induced the cleavage of caspase- 3 in these organoids (Fig. 6e). Importantly, infection with WT but not the cagA-deficient mutant $H$. pylori suppressed Raptinalinduced caspase- 3 activation in primary gastric epithelial cells (Fig. 6e). All together, these data demonstrate that H. pylori infection stimulates the expression cIAP2 in a CagA-dependent manner in human normal gastric epithelial cells, further supporting the notion that $H$. pylori infection confers apoptosis resistance in gastric epithelial cells.

\section{Discussion}

H. pylori infection-associated chronic inflammation and apoptosis resistance contribute to the development of gastric diseases, including gastritis and gastric cancer ${ }^{39}$. H. pylori-mediated inflammation is associated with its ability to activate NF- $\mathrm{kB}$ and the subsequent recruitment of co-activator Brd4 to the promoters and enhancers to facilitate NF- $\mathrm{kB}$-dependent inflammatory gene expres$\operatorname{sion}^{18}$. In this study, we found that Brd4 was also essential for $H$. pylori-induced NF-kB-dependent anti-apoptosis gene BIRC3 expression (Fig. 6e). By regulating BIRC3 eRNA and mRNA synthesis, Brd4 contributes to the development of apoptosis resistance in $H$. pylori-infected gastric epithelial cells (Fig. 6e).

We also showed that CagA was required for the $H$. pylori-induced cIAP2 expression and apoptosis resistance (Figs. 5 and 6). Since H. pylori CagA uses diverse pathways to activate NF- $\mathrm{kB}$, which regulates the expression of $\operatorname{cIAP2}^{14,19,32}$, it is possible that CagA utilizes activation of NF- $\mathrm{kB}$ to regulate the expression of BIRC3 eRNA and the production of cIAP2. In addition to its involvement in NF- $\mathrm{kB}$-dependent expression of cIAP2 for apoptosis resistance, CagA appears to utilize other strategies to inhibit cell apoptosis in infected cells. Injection of CagA protein into gastric epithelial cells has been 
A
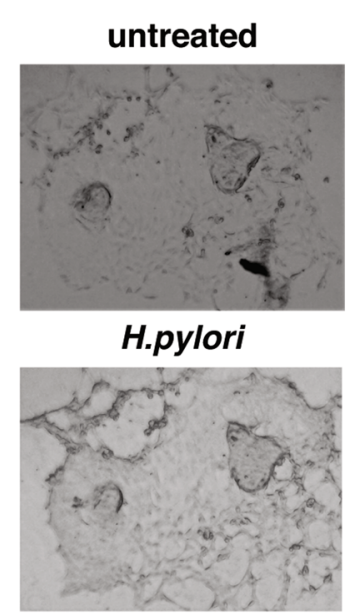

$\Delta$ CagA

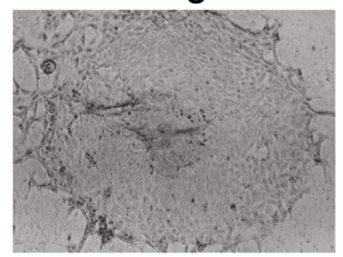

$-50 \mu \mathrm{m}$

D

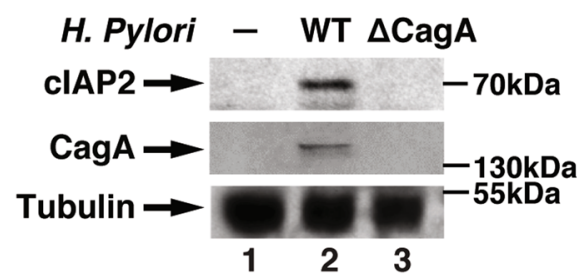

B

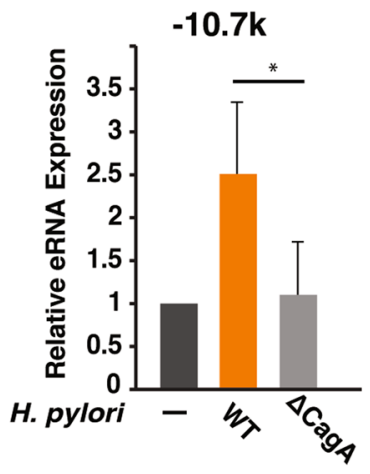

C

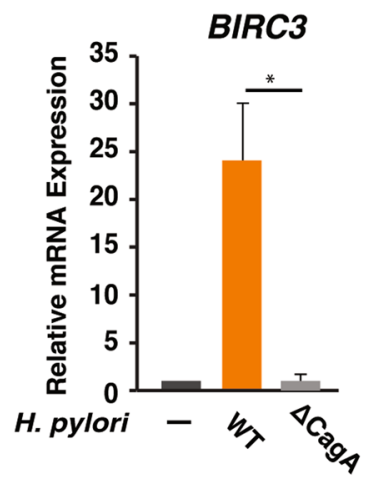

E

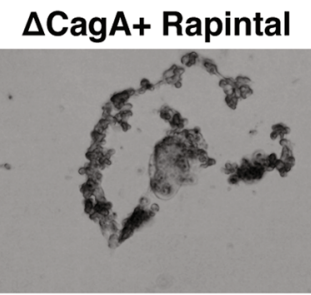

$\begin{aligned} \text { H. pylori } & - \\ \text { Raptinal } & -+-+-+\end{aligned}$

FL Casp-3 $\rightarrow-\infty-35 \mathrm{kDa}$

Cleaved

Casp-3 $\rightarrow \infty-17 \mathrm{kDa}$

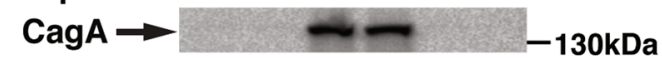

Tubulin $\rightarrow-\infty-5=-5 \mathrm{kDa}$

$\begin{array}{llllll}1 & 2 & 3 & 4 & 5 & 6\end{array}$

F

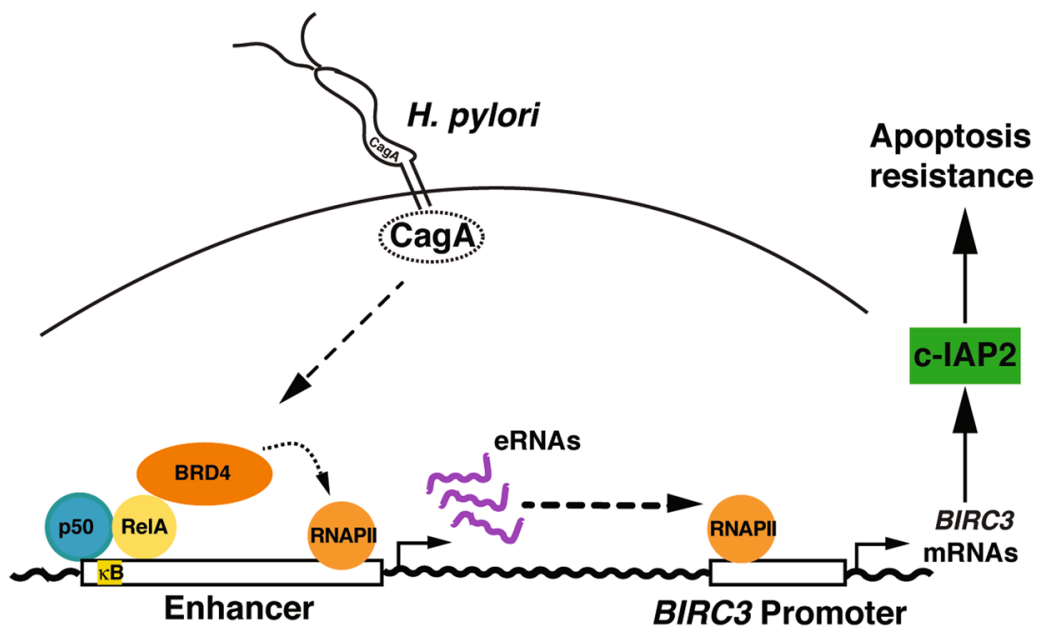

Fig. 6 (See legend on next page.) 
(see figure on previous page)

Fig. 6 H. pylori induces cIAP2 expression and inhibits apoptosis in primary human gastric epithelial cells. a Representative images of primary human gastric epithelial cells with or without infection by wild-type $\mathrm{H}$. pylori G27 or the cagA-deficient isogenic mutant for 1 h, followed by Raptinal treatment for $2 \mathrm{~h}$. Scale bar $=50 \mu \mathrm{m}$. b, c Primary human gastric epithelial cells were infected with $\mathrm{H}$. pylori G27 or cagA-deficient isogenic mutant for $1 \mathrm{~h}$. Levels of BIRC3 eRNA (b) or mRNA (c) were analyzed by qRT-PCR. d Primary human gastric epithelial cells were infected with $\mathrm{H}$. pylori G27 or cagA-deficient isogenic mutant for $8 \mathrm{~h}$. Cell lysates were immunoblotted for indicated proteins. (e). Primary human gastric epithelial cells were infected with $\mathrm{H}$. pylori G27 or cagA-deficient isogenic mutant for $1 \mathrm{~h}$ followed by the treatment with Raptinal $(10 \mu \mathrm{M})$ for $2 \mathrm{~h}$. Cell lysates were immunoblotted for indicated proteins. f Schematic model for the H. pylori infection-mediated CIAP2 induction and apoptosis resistance. H. pylori stimulates the recruitment of Brd4 to the enhancer of BIRC3 in a CagA-dependent manner. This recruitment facilitates RNAPII-mediated eRNA and mRNA synthesis and the induction of CIAP2, resulting in the inhibition of apoptosis. All data are shown as the mean \pm SD from three independent experiments. ${ }^{*} p<0.05$.

shown to counteract the apoptotic action of $H$. pylori VacA for the survival of $H$. pylori after colonization ${ }^{40}$. CagA-mediated pro-inflammatory cytokine expression and chronic inflammation could also provide a survival environment for infected gastric epithelial cells ${ }^{41}$. Furthermore, $H$. pylori CagA could activate ERK and antiapoptotic protein MCL1 in the gastric pits to suppress apoptosis or subvert the apoptosis-stimulating protein $\mathrm{p} 53^{42,43}$. How CagA coordinates these different cellular pathways to acquire the apoptosis resistance in $\mathrm{H}$. pyloriinfected cells remains to be further evaluated.

By activating the expression of cIAP2, $H$. pylori infection likely provides a survival advantage for the infected gastric epithelial cells, resulting in the enhanced colonization to establish long-term infection. Intriguingly, H. pylori can also induce epithelial cell apoptosis after prolonged infection. $H$. pylori activates the release of cytochrome $\mathrm{c}$ for the activation of caspase- 8 and the apoptosis after $12 \mathrm{hr}$ infection ${ }^{44}$. The induction of cIAP2 expression could be as early as $1 \mathrm{~h}$ after $H$. pylori infection (Fig. 2). The cell apoptosis after extended infection might result from reduced cIAP2 expression mediated by some negative feedback regulators of NF- $\mathrm{kB}$ (Fig. 5c). In this regard, $H$. pylori has been reported to activate A20, an inhibitor of NF-kB, to suppress NF-kB-mediated antiapoptotic effect ${ }^{45}$. H. pylori infection could also induce cell death in macrophages by polyamine-dependent mechanisms and by signaling via ERK-MAPK pathway, enabling the $H$. pylori to escape immune cells for persistent infection ${ }^{46-48}$. It has to be noted that $H$. pylori has been shown to sensitize TRAIL- and FasL-mediated apoptosis in gastric epithelial cells ${ }^{49,50}$, although $H$. pylori infection conferred resistance to Raptinal-induced apoptosis (Fig. 1). It appears that H. pylori infection differentially affects the intrinsic and the extrinsic apoptosis pathways. Therefore, H. pylori infection-mediated cell death or cell survival depends on the cell types and the apoptosis pathways.

H. pylori has been shown to regulate the expression of cIAP2 by activating the promoter of $B I R C 3$ via NF- $\mathrm{kB}$ in a luciferase reporter assay ${ }^{14}$. In addition to the promotermediated transcription, our current study demonstrated that BIRC3 enhancer was actively involved in the transcription of BIRC3 mRNA. H. pylori stimulated the synthesis of enhancer-derived eRNAs, which were critical for the transcription of BIRC3 mRNA and the production of cIAP2 (Fig. 3). eRNAs are regulatory non-coding RNAs that could regulate cis localized mRNA expression in various cell types ${ }^{25,26}$. eRNAs regulate gene transcription via enhancing the interaction between enhancers and promoters or facilitating the RNAPII recruitment and activation on the promoters of target genes ${ }^{24,26}$. Consistent with eRNA's role in gene transcription, inhibition of the BIRC3 eRNA synthesis abolished BIRC3 mRNA synthesis and cIAP2 production (Fig. 3). Importantly, inhibition of eRNA synthesis also impaired $H$. pylorimediated inhibition of caspase- 3 activation in gastric epithelial cells (Fig. 3f), suggesting that modulation of BIRC3 eRNA synthesis could dictate the survival and death outcome in $H$. pylori infected cells. It appears that cIAP2 is the major IAPs responsible for $H$. pylori-induced apoptosis resistance since the expression of BIRC3 but not BIRC2 and BIRC4 was significantly upregulated by H. pylori infection (Fig. 2a, c) and inhibition of cIAP2 was sufficient to eliminate $H$. pylori infection-mediated apoptosis resistance (Fig. 2f). However, cIAP1 and XIAP might also contribute to $H$. pylori infection-associated apoptosis resistance since cIAP1, cIAP2, and XIAP tend to form stable IAP complexes to more efficiently block the activation of caspases ${ }^{11}$.

H. pylori infection stimulated the recruitment of Brd4 to the active BIRC3 enhancer, which is associated with H3K27Ac marks (Fig. 4i). More importantly, Brd4 cooccupied with RelA and RNAPII on the eRNA-producing BIRC3 enhancer and was responsible for $H$. pyloriinduced BIRC3 eRNA synthesis (Fig. 4i). The recruitment of Brd4 and RNAPII to BIRC3 enhancer likely relies on the binding of NF- $\mathrm{BB}$ to the enhancer since NF- $\mathrm{KB}$ is known to assist the recruitment of Brd4 and RNAPII to the enhancer regions of many genes ${ }^{18}$. Brd4 has emerged as a therapeutic target in cancer and inflammatory diseases, and there are many clinical trials with different BETi, including JQ1, for their efficacies against tumors and inflammation ${ }^{51,52}$. In this study, we demonstrated 
that JQ1 increased the sensitivity of gastric epithelial cells to Raptinal-induced apoptosis in $H$. pylori-infected cells (Fig. 4). In the presence of JQ1, $H$. pylori barely activated the expression of cIAP2 and failed to suppress Raptinalinduced caspase-3 activation (Fig. 4). Therefore, future studies might be needed to test whether JQ1-like small molecules could be potentially used in combinational therapy to increase the sensitivity of cancer cells to chemotherapy or reduce the chemoresistance by downregulating the expression of anti-apoptosis genes.

Gastric organoids have emerged as a model system to study $H$. pylori pathogenesis ${ }^{37}$. In addition to the gastric cancer epithelial cells, we found that $H$. pylori also activated the expression of BIRC3 eRNA and mRNA and suppressed Raptinal-induced caspase- 3 activation in human gastric organoids (Fig. 6). Therefore, the mechanism we identified in this study for $H$. pylori infectionmediated apoptosis resistance might indeed represent the infected epithelial cells in vivo. Consistent with this, we found that the expression of BIRC3 was enhanced in the stomach of $H$. pylori-infected mice, and the expression was inhibited by the treatment of JQ1 (data not shown).

In summary, we have identified a novel function of Brd4 in H. pylori-induced apoptosis resistance. Brd4 was critically involved in $H$. pylori-mediated apoptosis resistance by inducing the production of cIAP2 via BIRC3 eRNA synthesis. Inhibition of Brd4 or elimination of Brd4mediated BIRC3 eRNA synthesis suppressed infectionassociated apoptosis resistance. Many anti-cancer drugs induce cancer cell apoptosis during chemotherapy and chemoresistance often occurs due to the development of apoptosis resistance ${ }^{53}$. Identification of Brd4 as a novel regulator of $\mathrm{H}$. pylori-induced apoptosis resistance provides not only new insights into the pathogenesis of H. pylori infection but also provides potential alternative approaches by targeting Brd4 for the prevention and treatment of $H$. pylori-associated gastric cancer and drugresistance. In addition to $H$. pylori, various pathogens, including Chlamydophila pneumonia, Neisseria gonorrhoeae and influenza virus, induce cIAP2 expression for the apoptosis resistance and persistent infection ${ }^{13,54,55}$. It would be of great interest to determine whether inhibition of Brd4 could be a general approach to eliminate pathogen-infection-associated apoptosis resistance.

\section{Materials and methods}

\section{Cell lines, reagents, and antibodies}

Human gastric cancer cell lines AGS, MKN28, MKN45 were cultured in RPMI-1640 medium supplemented with $10 \%$ fetal bovine serum; primary human gastric organoids were prepared and cultured as previously described ${ }^{56}$. Raptinal was a gift from Dr. Hergenrother (UIUC). BV6 was kindly provided by Dr. Vucic (Genentech, Inc). JQ1 has been described previously ${ }^{57}$. Cisplatin was purchased from Sigma. Lipofectamine ${ }^{\circledR}$ RNAiMAX Transfection was purchased from Thermo Fisher. Antibodies against cIAP1 (sc-7943), RNAPII (sc-899), RelA (sc-372), CagA (sc-25766), Brd3 (sc-81202) were purchased from Santa Cruz Biotechnology; antibodies against caspase-3 (\#9662), Brd2 (\#5848), cIAP2 (\#3130), XIAP (\#14334), Survivin (\#2808) were purchased from Cell Signaling Technology; antibodies against $\beta$-Tubulin (T5168) were purchased from Sigma; antibodies against H3K27ac (ab4729) were purchased from Abcam.

\section{H. pylori strains, H. pylori culture and infection}

H. pylori G27, a cagPAI (cag pathogenicity island)positive and virulence factor CagA-positive clinical isolate, and its isogeneic cagA-deficient strain have been previously described ${ }^{58}$. H. pylori was cultured in bisulphite-free Brucella broth supplemented with $10 \%$ fetal bovine serum and $5 \mu \mathrm{g} / \mathrm{ml}$ vancomycin at $37^{\circ} \mathrm{C}$ in the presence of $10 \% \mathrm{CO}_{2}$. H. pylori cultured overnight was added to AGS or primary human gastric epithelial cells for infection at a multiplicity of infection of 100 .

\section{siRNA knockdown, immunoblotting, and quantitative real- time PCR analysis}

siRNAs targeting BIRC3 (hs.Ri.BIRC3.13.1, hs.Ri. BIRC3.13.2), Brd2 (hs.Ri.Brd2.13.2, hs.Ri.Brd2.13.3) and Brd3 (hs.Ri.Brd3.13.1, hs.Ri.Brd3.13.2) and negative control siRNA (Cat\#51-01-14-03) were purchased from IDT; siRNAs targeting Brd4 (Cat\#4427038, ID: S23901/ S23902) were purchased from Ambion; siRNAs targeting BIRC3 eRNA were designed and purchased from Dharmacon, sequences for the duplexes are No.1: GGAGAAAGAAUCUGUUUUAUU; UAAAACAGAUU CUUUCUCCUU, No.2: CAAGUUGGAUUGAACAU CAUU; UGAUGUUCAAUCCAACUUGUU. All siRNAs were transfected using Lipofectamine RNAiMAX Transfection Reagent (Invitrogen) according to the manufacturer's protocol. Twenty-four hr post-transfection, cells were used for various experiments. Immunoblotting and quantitative real-time PCR analysis were performed as previously described ${ }^{21}$. All the western blots are representative images of at least three independent experiments. PCR primers for mRNA and eRNAs were purchased from Integrated DNA Technologies and the sequences of primers are provided in the Supplementary Information.

\section{ChIP-seq analysis}

ChIP-seq datasets were obtained from GSE85467 and GSE75595. Reads were mapped to human genome (GRCh37/hg19) using bowtie2 ${ }^{59}$. Peaks were identified using HOMER findPeaks program and annotated using anotatePeaks.pl ${ }^{60}$. Finally, the resulting data were uploaded to UCSC genome browser to obtain the visual mapping of each histone modification ${ }^{61}$. 


\section{Chromatin immunoprecipitation assays (ChIPs)}

ChIP assay was performed as previously described ${ }^{57}$. In brief, 10 to 20 million cells were fixed in $1 \%$ formaldehyde for $10 \mathrm{~min}$ and sonicated using a bioruptor (Cole-Parmer) to obtain DNA peak length at $500 \mathrm{bp}$, and lysates were immunoprecipitated overnight with various antibodies. Protein A agarose blocked with sheared salmon sperm DNA, was used to collect antibody-chromatin complexes. After reverse crosslink with proteinase K, DNA was extracted with DNA purification kit from Qiagen (Valencia, CA, USA). The sequence of ChIP primers are: $-10.7 \mathrm{k}$ forward: AAGCTACCTCTCAGCCTACTTT; $-10.7 \mathrm{k}$ reverse: CCACTGTTTTCTGTACCCGGA.

\section{Flow cytometry}

Cell death was analyzed by fluorescence activated cell sorting with FITC-conjugated annexin $\mathrm{V}$ and propidium iodide (BD Bioscience) according to the manufacturer's instructions. In brief, the cells were collected and washed followed by incubation with annexin V and PI for $15 \mathrm{~min}$. Cells then were analyzed using BD LSR II flow cytometer. Flowjo software was used to analyze the data.

\section{Statistical analysis}

Data analysis was carried out using GraphPad Prism version 5 (GraphPad Software, San Diego California USA). All data are presented as mean \pm SD unless otherwise stated. Student unpaired $t$-test was used to analyze the data. For all data, $p$ value $\leq 0.05$ was considered statistically significant. ${ }^{*} p<0.05,{ }^{* * *} p<0.01$, and ${ }^{* * * *} p<0.005$.

\section{Acknowledgements}

We thank members of the Chen lab for discussion. We thank Dr. Hergenrother for kindly providing Raptinal and Dr. Vucic for BV6. This work is supported in part by fund provided by UIUC and Keck Foundation.

\section{Author details}

${ }^{1}$ Department of Biochemistry, University of Illinois at Urbana-Champaign, Urbana 61801 IL, USA. ${ }^{2}$ Fujian Key Laboratory for Translational Research in Cancer and Neurodegenerative Diseases, Institute for Translational Medicine, School of Basic Medical Sciences, Fujian Medical University, Fuzhou 350122, China. ${ }^{3}$ The State Key Laboratory of Cellular Stress Biology, School of Life Sciences, Xiamen University, Xiamen 361101, China. ${ }^{4}$ Present address: Department of Pharmacology and Systems Physiology, University of Cincinnati, Cincinnati 45267 OH, USA. ${ }^{5}$ Present address: Division of Gastroenterology, Department of Medicine and Cancer Biology, Vanderbilt University School of Medicine, Nashville 37232 TN, USA. ${ }^{6}$ Present address: Department of Cellular and Molevular Medicine, College of Medicine, University of Arizona-Tucson, Tucson 85724 AZ, USA. ${ }^{7}$ Present address: Carle R. Woese Institute for Genomic Biology, University of Illinois at Urbana-Champaign, Urbana 61801 IL, USA

\section{Conflict of interest}

The authors declare that they have no conflict of interest.

\section{Publisher's note}

Springer Nature remains neutral with regard to jurisdictional claims in published maps and institutional affiliations.

Supplementary Information accompanies this paper at (https://doi.org/ 10.1038/s41419-020-02894-z)
Received: 25 March 2020 Revised: 28 July 2020 Accepted: 29 July 2020 Published online: 21 August 2020

\section{References}

1. Faherty, C. S. \& Maurelli, A. T. Staying alive: bacterial inhibition of apoptosis during infection. Trends Microbiol 16, 173-180 (2008).

2. Peek, R. M. Jr. \& Blaser, M. J. Helicobacter pylori and gastrointestinal tract adenocarcinomas. Nat. Rev. Cancer 2, 28-37 (2002).

3. Hatakeyama, M. Oncogenic mechanisms of the Helicobacter pylori CagA protein. Nat. Rev. Cancer 4, 688-694 (2004).

4. Parsonnet, J., Friedman, G. D., Orentreich, N. \& Vogelman, H. Risk for gastric cancer in people with CagA positive or CagA negative Helicobacter pylori infection. Gut 40, 297-301 (1997).

5. Blaser, M. J. et al. Infection with Helicobacter pylori strains possessing cagA is associated with an increased risk of developing adenocarcinoma of the stomach. Cancer Res. 55, 2111-2115 (1995).

6. Ohnishi, N. et al. Transgenic expression of Helicobacter pylori CagA induces gastrointestinal and hematopoietic neoplasms in mouse. Proc. Natl Acad. Sci. USA 105, 1003-1008 (2008).

7. Shalini, S., Dorstyn, L., Dawar, S. \& Kumar, S. Old, new and emerging functions of caspases. Cell Death Differ. 22, 526-539 (2015).

8. Kumar, S. Caspase function in programmed cell death. Cell Death Differ. 14 32-43 (2007).

9. Fulda, S. \& Debatin, K. M. Extrinsic versus intrinsic apoptosis pathways in anticancer chemotherapy. Oncogene 25, 4798-4811 (2006).

10. Palchaudhuri, R. et al. A small molecule that induces intrinsic pathway apoptosis with unparalleled speed. Cell Rep. 13, 2027-2036 (2015).

11. Gyrd-Hansen, M. \& Meier, P. IAPs: from caspase inhibitors to modulators of NFkappaB, inflammation and cancer. Nat. Rev. Cancer 10, 561-574 (2010).

12. Rajalingam, $\mathrm{K}$. et al. IAP-IAP complexes required for apoptosis resistance of $C$. trachomatis-infected cells. PLoS Pathog. 2, e114 (2006).

13. Binnicker, M. J., Williams, R. D. \& Apicella, M. A. Infection of human urethra epithelium with Neisseria gonorrhoeae elicits an upregulation of host antiapoptotic factors and protects cells from staurosporine-induced apoptosis. Cell Microbiol 5, 549-560 (2003).

14. Yanai, A. et al. Helicobacter pylori induces antiapoptosis through buclear factorkappaB activation. J. Infect. Dis. 188, 1741-1751 (2003).

15. Li, Z., Chen, J., Chan, K. W., Qiao, L. \& Wong, B. C. A possible role of CIAP2 in Helicobacter pylori-associated gastric cancer. Cancer Lett. 313, 192-200 (2011).

16. Chang, C. S., Chen, W. N., Lin, H. H., Wu, C. C. \& Wang, C. J. Increased oxidative DNA damage, inducible nitric oxide synthase, nuclear factor kappaB expression and enhanced antiapoptosis-related proteins in Helicobacter pylori-infected non-cardiac gastric adenocarcinoma. World J. Gastroenterol. 10, 2232-2240 (2004).

17. Chen, L. F. \& Greene, W. C. Shaping the nuclear action of NF-kB. Nat. Rev. Mol. Cell Biol. 5, 392-401 (2004).

18. Chen, J. et al. BET inhibition attenuates Helicobacter pylori-induced inflammatory response by suppressing inflammatory gene transcription and enhancer activation. J. Immunol. 196, 4132-4142 (2016).

19. Lamb, A. \& Chen, L. F. The many roads traveled by Helicobacter pylori to NFkappaB activation. Gut Microbes 1, 109-113 (2010).

20. Smale, S. T. \& Natoli, G. Transcriptional control of inflammatory responses. Cold Spring Harb. Perspect. Biol. 6, a016261 (2014).

21. Huang, B., Yang, X. D., Zhou, M. M., Ozato, K. \& Chen, L. F. Brd4 coactivates transcriptional activation of NF-kappaB via specific binding to acetylated RelA. Mol. Cell Biol. 29, 1375-1387 (2009).

22. Brasier, A. R. et al. RelA Ser276 phosphorylation-coupled Lys310 acetylation controls transcriptional elongation of inflammatory cytokines in respiratory syncytial virus infection. J. Virol. 85, 11752-11769 (2011).

23. Hah, N. et al. Inflammation-sensitive super enhancers form domains of coordinately regulated enhancer RNAs. Proc. Natl Acad. Sci. USA 112 E297-E302 (2015)

24. Kaikkonen, M. U. et al. Remodeling of the enhancer landscape during macrophage activation is coupled to enhancer transcription. Mol. Cell 51, 310-325 (2013).

25. Lam, M. T., Li, W., Rosenfeld, M. G. \& Glass, C. K. Enhancer RNAs and regulated transcriptional programs. Trends Biochem Sci. 39, 170-182 (2014).

26. NE, I. I. et al. Long non-coding RNAs and enhancer RNAs regulate the lipopolysaccharide-induced inflammatory response in human monocytes. Nat. Commun. 5, 3979 (2014). 
27. Varfolomeev, E. et al. IAP antagonists induce autoubiquitination of C-IAPS, NFkappaB activation, and TNFalpha-dependent apoptosis. Cell 131, 669-681 (2007).

28. Spicuglia, S. \& Vanhille, L. Chromatin signatures of active enhancers. Nucleus $\mathbf{3}$, 126-131 (2012).

29. Guil, S. \& Esteller, M. Cis-acting noncoding RNAs: friends and foes. Nat. Struct. Mol. Biol. 19, 1068-1075 (2012).

30. Khan, Y. M., Kirkham, P., Barnes, P. J. \& Adcock, I. M. Brd4 is essential for IL1 beta-induced inflammation in human airway epithelial cells. PLOS ONE 9 e95051 (2014).

31. Filippakopoulos, P. et al. Selective inhibition of BET bromodomains. Nature 468, 1067-1073 (2011).

32. Backert, S. \& Naumann, M. What a disorder: proinflammatory signaling pathways induced by Helicobacter pylori. Trends Microbiol. 18, 479-486 (2010).

33. Shibata, W. et al. CagA protein secreted by the intact type IV secretion system leads to gastric epithelial inflammation in the Mongolian gerbil model. J. Pathol. 210, 306-314 (2006).

34. Schumacher, M. A. et al. The use of murine-derived fundic organoids in studies of gastric physiology. J. Physiol. 593, 1809-1827 (2015).

35. Mahe, M. M. et al. Establishment of gastrointestinal epithelial organoids. Curr. Protoc. Mouse Biol. 3, 217-240 (2013).

36. Wroblewski, L. E. et al. Helicobacter pylori targets cancer-associated apicaljunctional constituents in gastroids and gastric epithelial cells. Gut 64, 720-730 (2015).

37. Pompaiah, M. \& Bartfeld, S. Gastric organoids: an emerging model system to study Helicobacter pylori pathogenesis. Curr. Top. Microbiol Immunol. 400, 149-168 (2017).

38. Bertaux-Skeirik, N. et al. CD44 plays a functional role in Helicobacter pylori-induced epithelial cell proliferation. PLoS Pathog. 11, e1004663 (2015).

39. Israel, D. A. \& Peek, R. M. pathogenesis of Helicobacter pylori-induced gastric inflammation. Aliment Pharm. Ther. 15, 1271-1290 (2001).

40. Oldani, A. et al. Helicobacter pylori counteracts the apoptotic action of its VacA toxin by injecting the CagA protein into gastric epithelial cells. PLoS Pathog. $\mathbf{5}$ e1000603 (2009).

41. Lee, K. E. et al. Helicobacter pylori and interleukin-8 in gastric cancer. World J. Gastroenterol. 19, 8192-8202 (2013).

42. Mimuro, $\mathrm{H}$. et al. Helicobacter pylori dampens gut epithelial self-renewal by inhibiting apoptosis, a bacterial strategy to enhance colonization of the stomach. Cell Host Microbe 2, 250-263 (2007).

43. Buti, L. et al. Helicobacter pylori cytotoxin-associated gene A (CagA) subverts the apoptosis-stimulating protein of p53 (ASPP2) tumor suppressor pathway of the host. Proc. Natl Acad. Sci. USA 108, 9238-9243 (2011).

44. Maeda, S. et al. Analysis of apoptotic and antiapoptotic signalling pathways induced by Helicobacter pylori. Gut 50, 771-778 (2002).
45. Lim, M. C. C. et al. Pathogen-induced ubiquitin-editing enzyme A20 bifunctionally shuts off NF-kappaB and caspase-8-dependent apoptotic cell death. Cell Death Differ. 24, 1621-1631 (2017).

46. Allen, L. A., Schlesinger, L. S. \& Kang, B. Virulent strains of Helicobacter pylori demonstrate delayed phagocytosis and stimulate homotypic phagosome fusion in macrophages. J. Exp. Med 191, 115-128 (2000).

47. Pathak, S. K., Tavares, R., de Klerk, N., Spetz, A. L. \& Jonsson, A. B. Helicobacter pylori protein JHP0290 binds to multiple cell types and induces macrophage apoptosis via tumor necrosis factor (TNF)-dependent and independent pathways. PLoS ONE 8, e77872 (2013).

48. Gobert, A. P. et al. Helicobacter pylori induces macrophage apoptosis by activation of arginase II. J. Immunol. 168, 4692-4700 (2002).

49. Martin, J. H. et al. Effect of H. pylori on the expression of TRAIL, FasL and their receptor subtypes in human gastric epithelial cells and their role in apoptosis. Helicobacter 9, 371-386 (2004).

50. Lin, W. C. et al. Helicobacter pylori sensitizes TNF-related apoptosis-inducing ligand (TRAIL)-mediated apoptosis in human gastric epithelial cells through regulation of FLIP. Cell Death Dis. 5, e1109 (2014).

51. Filippakopoulos, P. \& Knapp, S. Targeting bromodomains: epigenetic readers of lysine acetylation. Nat. Rev. Drug Discov. 13, 337-356 (2014).

52. Shi, J. \& Vakoc, C. R. The mechanisms behind the therapeutic activity of BET bromodomain inhibition. Mol. Cell 54, 728-736 (2014).

53. Shi, W. J. \& Gao, J. B. Molecular mechanisms of chemoresistance in gastric cancer. World J. Gastrointest. Oncol. 8, 673-681 (2016).

54. Paland, N. et al. NF-kappaB and inhibitor of apoptosis proteins are required for apoptosis resistance of epithelial cells persistently infected with Chlamydophila pneumoniae. Cell Microbio/ 8, 1643-1655 (2006).

55. Rodrigue-Gervais, I. G. et al. Cellular inhibitor of apoptosis protein CIAP2 protects against pulmonary tissue necrosis during influenza virus infection to promote host survival. Cell Host Microbe 15, 23-35 (2014).

56. Teal, E., Bertaux-Skeirik, N., Chakrabarti, J., Holokai, L. \& Zavros, Y. Establishment of human- and mouse-derived gastric primary epithelial cell monolayers from organoids. Methods Mol. Biol. 1817, 145-155 (2018).

57. Zou, Z. et al. Brd4 maintains constitutively active NF-kappaB in cancer cells by binding to acetylated RelA. Oncogene 33, 2395-2404 (2014).

58. Lamb, A. et al. Helicobacter pylori CagA activates NF-kappaB by targeting TAK1 for TRAF6-mediated Lys 63 ubiquitination. EMBO Rep. 10, 1242-1249 (2009).

59. Langmead, B. \& Salzberg, S. L. Fast gapped-read alignment with Bowtie 2. Nat. Methods 9, 357-359 (2012).

60. Heinz, S. et al. Simple combinations of lineage-determining transcription factors prime cis-regulatory elements required for macrophage and $B$ cell identities. Mol. Cell 38, 576-589 (2010).

61. Kent, W. J. et al. The human genome browser at UCSC. Genome Res $\mathbf{1 2}$ 996-1006 (2002). 